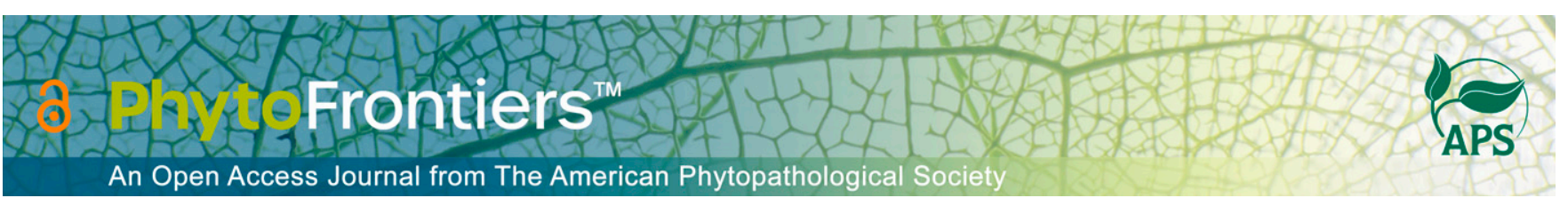

\title{
Research
}

\section{Isothiocyanate Derivatives of Glucosinolates as Efficient Natural Fungicides}

\author{
Sylvain Dubey ${ }^{1,2,3,+}$ (1) | Florian Guignard ${ }^{1,2} \mid$ Sébastien Pellaud ${ }^{1,2}$ | Matteo Pedrazzetti ${ }^{1,2}$ | \\ Alja van der Schuren ${ }^{1,2} \mid$ Alain Gaume ${ }^{2}$ Sylvain Schnee ${ }^{2}$ | Katia Gindro ${ }^{2}$ | Olga Dubey ${ }^{1,2}$ |
}

1 AgroSustain SA, CH-1260 Nyon, Switzerland

2 Agroscope, Swiss Federal Agricultural Research Station in Changins, $\mathrm{CH}-1260$ Nyon, Switzerland

${ }^{3}$ Department of Ecology and Evolution, University of Lausanne, 1015 Lausanne, Switzerland

† Corresponding author: S. Dubey; sylvain.dubey@ agrosustain.ch

Accepted for publication 21 September 2020.

\section{Funding}

The authors are grateful to Innosuisse (Swiss Innovation Agency), FIT (Fondation pour l'Innovation Technologique), SPEI (Service de la promotion de l'économie et de l'innovation), and the foundation Gebert Rüf Stiftung for funding.

The author(s) declare no conflict of interest.

$\boldsymbol{e}$-Xtra: One supplementary figure and two supplementary tables are available online.

\begin{abstract}
Fungal pathogens on crops account for losses that exceed US $\$ 200$ billion annually. At present, chemical fungicides are widely used in the agricultural industry. Many of these products have a detrimental effect on human and animal health and are consequently forbidden postharvest, especially in Europe. Despite efforts to develop natural crop protection, very few have been commercialized. We explored the physicochemical characteristics of (i) glucosinolate derivatives from the present study and previously published papers in the light of their known biological roles and (ii) fungitoxic glucosinolate derivatives compared with natural and chemical fungicides. We found that 13 out of 31 tested natural and semisynthetic isothiocyanates are efficient fungicides against widespread species of plant pathogens alone and in a synergistic manner. Interestingly, physicochemical characteristics of fungitoxic glucosinolate derivatives differ from those showing no activities or known for their insecticidal or insect-attractive properties. The comparison of physicochemical characteristics of natural and semisynthetic fungitoxic glucosinolate derivatives with other fungicides (natural, semisynthetic, and synthetic) revealed that isothiocyanate glucosinolate derivatives clustered with nonglucosinolate derivatives plant fungicides as well as with some synthetic ones. Most of the fungicides show high bioaccumulation potential and lipophilic properties that most likely allow them to go through membranes.
\end{abstract}

Keywords: isothiocyanate, glucosinolate derivatives, Brassicaceae, natural fungicide, synthetic fungicide, synergy, plant, fungi, porifera, bacteria

Food rotting in the field and further down the supply chain is an all-too-common sight. More disturbingly, the Food and Agriculture Organization estimates that approximately one-third of global food production - worth around US\$1.66 trillion-goes to waste annually (FAO 2011). Fungal pathogens on perishable foods such as fruits and vegetables during pre- and postharvest phases are estimated to account for losses that exceed US\$200 billion annually (Gonzalez-Fernandez et al. 2010). 
At present, chemical fungicides are widely used in the agricultural industry. Many of these products have a detrimental effect on human and animal health and are consequently forbidden postharvest, especially in Europe (Lucas et al. 2015). Despite efforts to develop natural protection, very few efficient solutions have been commercialized, and there are still no effective environmentally friendly methods of combatting the fungal pathogens that infect large groups of crops (Yoon et al. 2013).

Compounds with antifungal activities can be found in various organisms from prokaryotes to eukaryotes (e.g., El Amraoui et al. 2014; Hadizadeh et al. 2015), such as plants, with oil mixtures containing terpenes, phenols, and alcohols (Ahmad et al. 2010; Cortés-Rojas et al. 2014; Tang et al. 2018), glycosides (Yoon et al. 2011), alkaloids (Yoon et al. 2013), and recently discovered glucosinolate derivatives (GLSD; Dubey et al. 2020). These latter compounds are primarily present in the family of Brassicaceae and in some families of the order Brassicales (Fahey et al. 2001; Yamane et al. 2010). They are the results of myrosinase enzyme breaking down glucosinolates (GLSs; a well-defined group of plant metabolites characterized by an $S$ - $\beta$-D-glucopyrano unit connected to an $O$-sulfated ( $Z$ )-thiohydroximate function; Blažević et al. 2020) during mechanical tissue damage (e.g., by herbivores) into three major families of bioactive compounds: nitriles, thiocyanates, and isothiocyanates. The system in which myrosinase and GLS enter into contact when tissues are damaged is called the glucosinolate-myrosinase system (Ishida et al. 2014). Indeed, myrosinase is distributed in myrosin cells that differ to cells containing GLS, preventing a breakdown of GLS in the absence of tissue damage (Chhajed et al. 2019). The proportion of each derivative resulting from the enzymatic hydrolysis of GLS strongly depends on factors such as temperature and $\mathrm{pH}$ and is also species specific (Cartea and Velasco 2008; Hanschen et al. 2017). GLSs and hence their derivatives are a very diverse group, and their polymorphism is frequent in plant species as well as among different organs (Agerbirk and Olsen 2012; Ishida et al. 2014).

The roles of GLSD in plants are not fully understood, as well as the evolutionary driving forces leading to such a diversity of compounds. Some were shown to be involved in defense responses against bacteria and herbivores (Wittstock et al. 2003), but fungitoxic activities remain poorly documented (Dubey et al. 2020). In addition, specialist herbivorous species (e.g., cabbage white butterflies) are attracted by plants containing glucosinolate derivatives as well as parasitoid wasps targeting these herbivores (van Poecke et al. 2001; Wittstock et al. 2003). Finally, herbivores such as cabbage aphids sequester these compounds from host plants as a defense against predators (Kos et al. 2012; Vanhaelen et al. 2002).

Dubey et al. (2020) highlighted that 7-methylsulfonyl-heptyl isothiocyanate (compound 1; see Figure 1 and Supplementary Table S1), a breakdown product of 7-methylsulfonylheptyl glucosinolate, which overaccumulated on the plant surface of Arabidopsis thaliana polyunsaturated fatty acid (PUFA) mutants, has antifungal properties on widespread pathogenic fungal species. Based on these results, we investigated the fungitoxic activity of natural isothiocyanate derivatives of GLSs (methylsulfonyl and methylsulfinyl isothiocyanates) as well as semisynthetic derivatives and their synergy and compared it with semisynthetic methylsulfonyl and methylsulfinyl nitrile and thiocyanates. We also explored the physicochemical characteristics of (i) GLSD from this study and previously published papers in the light of their known biological roles (e.g., fungicide, insecticide, attractive to insects) and (ii) fungitoxic GLSD compared with natural and chemical fungicides.

\section{MATERIALS AND METHODS}

\section{Description of natural and semisynthetic compounds}

Methylsulfonyl and methylsulfinyl nitriles (10 compounds: four natural and six semisynthetic) and thiocyanates (eight compounds: four natural and four semisynthetic) were obtained from SpiroChem (Basel, Switzerland); methylsulfonyl and methylsulfinyl isothiocyanates (31 compounds: 16 natural and 15 semisynthetic) were obtained from SpiroChem and LKT Labs (St. Paul, MN).

The full IUPAC chemical names of the 49 compounds used in this study is given below. The natural and semisynthetic nitriles included, respectively, 4-(methylsulfinyl)butanenitrile, 7-(methanesulfonyl)heptanenitrile, 8-(methylsulfinyl)octanenitrile, and 8-(methylsulfonyl)octanenitrile and 2-((5-methylsulfinyl)pentyl) oxy)acetonitrile, 2-((5-methylsulfonyl)pentyl)oxy)acetonitrile, 2 (2-(2-(methylsulfinyl)ethoxy)ethoxy)acetonitrile, 2-(2-(2-(methylsulfonyl)ethoxy)ethoxy)acetonitrile, 3-(4-(methylsulfonyl)butoxy) propanenitrile, and 3-(4-(methylsulfinyl)butoxy)propanenitrile. Natural and semisynthetic thiocyanates included, respectively, 1-(methylsulfinyl)-4-thiocyanatobutane, 1-(methylsulfonyl)-4-thiocyanatobutane, 1-(methylsulfinyl)-8-thiocyanatooctane, and 1-(methylsulfonyl)-8-thiocyanatooctane and 1-(methylsulfinyl)-2-(2-(2-thiocyanatoethoxy)ethoxy)ethane, 1-(methylsulfonyl)-2-(2-(2-thiocyanatoethoxy)ethoxy)ethane, 1-(3-(methylsulfinyl)propoxy)-4-thiocyanatobutane, and 1-(3-(methylsulfonyl)propoxy)-4-thiocyanatobutane. Finally, natural and semisynthetic isothiocyanates included, respectively, 1-isothiocyanato-3-(methylsulfinyl)-propane, 1-isothiocyanato-3-(methylsulfonyl)-propane, 1-isothiocyanato-6-(methylsulfinyl)-hexane, 1-isothiocyanato-7-(methylsulfinyl)-heptane, 1isothiocyanato-8-(methylsulfinyl)-octane, 1-isothiocyanato-8-(methylsulfonyl)-octane, 1-isothiocyanato-9-(methylsulfinyl)-nonane, 1-isothiocyanato-9-(methylsulfonyl)-nonane, 1-isothiocyanato-4-(methylsulfinyl)-butane, 1-isothiocyanato-5-(methylsulfinyl)-pentane, 1-isothiocyanato-4-(methylsulfonyl)-butane, 1-isothiocyanato-5-(methylsulfonyl)-pentane, 1-isothiocyanato-6-(methylsulfonyl)-hexane, 1-isothiocyanato-7-(methylsulfonyl)-heptane, 1-isocyanato-4-(methylsulfonlyl)-butane, and 1-isothiocyanato-7-(methylsulfanyl)-heptane and (E)-1-isothiocyanato-8-(methylsulfinyl)oct-2-ene, 1-(ethylsulfinyl)8-isothiocyanatooctane, 1-(ethylsulfonyl)-8-isothiocyanatooctane, 1-(isothiocyanatomethyl)-3-(4-(methylsulfinyl)butyl)benzene, 1-(isothiocyanatomethyl)-3-(4-(methylsulfonyl)butyl)benzene, 1-isocyanato4-(methylsulfonyl)butane, 1-isocyanato-10-(methylsulfonyl)decane, ((8-isothiocyanatooctyl)sulfinyl)benzene, ((8-isothiocyanatooctyl) sulfonyl)benzene, (E)-1-isothiocyanato-8-(methylsulfonyl)oct-2ene, 1-isothiocyanato-4-(3-(methylsulfinyl)propoxy)butane, 1-isothi-o cyanato-4-(3-(methylsulfonyl)propoxy)butane, 1-(3-isothiocyanatopropyl)-4-((methylsulfinyl)methyl)benzene, 1-(3-isothiocyanatopropyl)-4-((methylsulfonyl)methyl)benzene, and 1-isothiocyanato-2-(2-(2-(methylsulfonyl)ethoxy)ethoxy)ethane.

All the obtained compounds were dissolved in dimethyl sulfoxide (DMSO) at $1 \mathrm{M}$ to make a stock solution. Fungal species used for in vitro fungal bioassays (described in the next section) were obtained from the mycotheca of Agroscope (www. mycoscope.bcis.ch). Final concentrations of DMSO used in biotests are not toxic to fungal pathogens and do not exceed $0.085 \%$ (see Supplementary Fig. S1).

\section{Biotests with single compounds and combinations}

We tested the fungitoxic activities of single and combined methylsulfonyl and methylsulfinyl nitriles and thiocyanates, methylsulfonyl and methylsulfinyl isothiocyanates, and one methylsulfanyl on three necrotrophic widespread fungal species 
causing severe food losses (that can easily be grown in laboratory conditions), from different orders and targeting both monocotyledons and dicotyledons: Alternaria radicina (class: Dothideomycetes; order: Pleosporales; host: carrot), Fusarium graminearum (class: Sordariomycetes; order: Hypocreales; host: cereals), and Plectosphaerella cucumerina (class: Sordariomycetes; order: Glomerellales; host: tomato and cucurbits).
Overall, a total of 133 combinations were tested on the three fungal species.

Fungitoxic activities were tested in 48-well tissue culture plates. In more detail, plant fungal pathogens were inoculated on potato dextrose agar complemented with isothiocyanates, nitriles, and thiocyanates that were preliminarily dissolved to various concentrations $(0,10,150,250,375,500,700$, and $850 \mu \mathrm{M})$ in
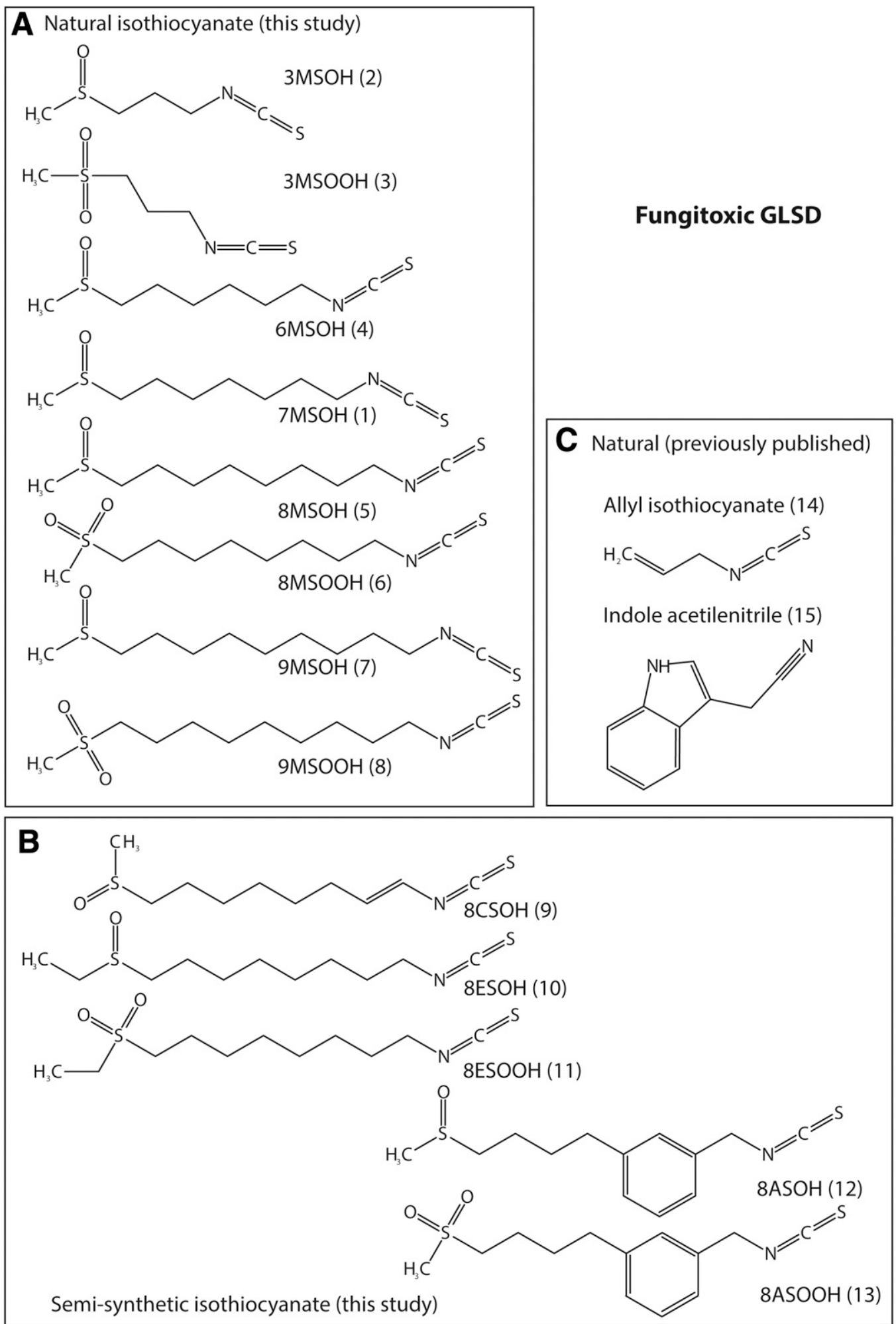

FIGURE 1

Structural formulas of natural (A) and semisynthetic isothiocyanates (B) showing fungitoxic activities (this study) and previously published natural fungitoxic glucosinolate derivatives (GLSD) (C). Compound numbers are indicated in parentheses. 
potato dextrose broth. Then 2-mm agar plugs (Dufour et al. 2015) of a fungal preculture were placed in each well, and three biological replicates were used for each concentration. Plates were incubated for a period of 1 week in the phytotron $(80 \%$ relative humidity, constant temperature of $23^{\circ} \mathrm{C}$, under alternating 16-h day and 8-h night cycles). Mycelial growth was measured after 7 days using ImageJ (http://imagej.net/Welcome). Synergistic fungitoxic activities were then estimated with CompuSyn software (www.combosyn.com; Chou 2006; Chou and Martin 2005). It was used to determine the combination index (CI) for combinations of two molecules and hence the presence of synergism $(\mathrm{CI}<1$; with values between 0.1 and 0.3 considered as strong synergism and values $<0.1$ as very strong synergism; Chou 2008).

\section{Physicochemical properties of GLS derivatives and known roles}

We performed a principal component analysis (PCA) to visualize how GLSD (fungicide, insecticide, attractive to insects, general toxicity, phytotoxicity, or with other functions/applications or unknown functions) differ from each other, based on their physicochemical characteristics (this study; Dubey et al. 2020; Lambrix et al. 2001; Wittstock et al. 2003; Supplementary Table S1). A total of eight variables, commonly used to describe properties of fungicides, were included in the analyses: molecular weight (MW, Dalton), boiling point $\left(\mathrm{BP},{ }^{\circ} \mathrm{C}\right)$, polar surface area (PS, surface sum over all polar atoms, $\AA^{2}$ ), Henry's law volatility constant $(\mathrm{HC}$, unitless), vapor pressure (VP, mmHG), log Koa (log octanol/air partition coefficient), log Kow (log octanol/water partition coefficient), and $\log \mathrm{S}$ (log of intrinsic solubility in mol/liter). Values for each parameter (except MW and PS) were estimated with Estimation Programs Interface software (U.S. EPA 2020).

We also tested for physicochemical property differences between attractive, insecticidal, and antifungal GLSD with ANOVA (tested variables are the same as in the PCA). Differences between categories (attractive, insecticidal, and antifungal GLSD) were determined by Tukey's honestly significant difference (HSD) test. In addition, a discriminant analysis was performed with theses variables in order to assess if they can be used to attribute compounds to well-defined groups (fungicide, insecticide, attractive). Finally, we tested for physicochemical differences between compounds (tested in this study) showing antifungal properties or not with ANOVAs.

All the analyses were performed with JMP (version 14. SAS Institute, Cary, NC, 1989 to 2019). Levels of significance were Bonferroni corrected for multiple tests (Rice 1989).

\section{Physicochemical properties of antifungal GLS derivatives versus other natural and synthetic fungicides}

A PCA was performed with fungitoxic GLSD (15 compounds), various natural and semisynthetic compounds (54 compounds; described from plant and algae [13], bacterial [8], fungal [19], and poriferan [14] origins from literature review and the Fungal Resistance Action Committee [FRAC 2019] database), and synthetic fungicides (65 compounds; from FRAC [2019] database, including all the known modes of action) to visualize how fungitoxic GLSD differ from these compounds, based on their physicochemical characteristics (we included the same variables as in the PCA performed strictly with GLSD; see Supplementary Table S2 for a complete list of fungicides used for the analysis).

We also tested for physicochemical differences between fungitoxic GLSD and other natural and synthetic fungicides with
ANOVA (tested variables are the same as in the PCA). Levels of significance were Bonferroni corrected for multiple tests (Rice 1989).

\section{RESULTS}

\section{Efficacy of tested compounds and synergy}

A total of seven single compounds (all isothiocyanates) out of the 49 tested showed fungitoxic activities at the tested concentrations (up to $850 \mu \mathrm{M}$ ): four natural and three semisynthetic compounds, respectively compounds $1,2,3$, and 5 and compounds 9, 12, and 13 (Fig. 1). Six additional tested compounds (all isothiocyanates) revealed fungitoxic activities in a synergistic manner when combined with some of the compounds above (i.e., 4, 6, 7, 8, 11, and 12, with the last two being semisynthetic) (Fig. 1, Table 1). Hence, only some isothiocyanate GLSD revealed antifungal properties and no nitrile or thiocyanate GLSD.

The results obtained for the synergistic combinations for each fungal species are summarized in Table 1. It shows the EC50 (range: 10 to $850 \mu \mathrm{M}$ ) obtained for the two molecules alone, for the combination of them (always smaller), and the lower CI. Because all CIs listed in the table are lower than 0.26, it indicates that the combined molecules have strong synergisms (Chou 2008).

\section{Physicochemical properties of GLS derivatives and known roles}

The PCA highlighted that GLSD with known functions exhibit different physicochemical characteristics (Fig. 2). The two first principal components explained $70.5 \%$ of the variance $(\mathrm{PC} 1$ : 48.3\%; PC2: $22.2 \%$ ). The discriminant analysis based on attractive, insecticidal, and antifungal GLSD confirms this result, with only one compound misclassified out of 28 (3.6\%; entropy $r^{2}$ : 0.83; -2 log likelihood: 9.5).

After Bonferroni correction (significance level: 0.00625), ANOVAs revealed significant differences between attractive, insecticidal, and antifungal GLSD for MW $\left(P<0.001 ; \mathrm{F}_{2,27}=\right.$ 36.43), PS $\left(P<0.001 ; \mathrm{F}_{2,27}=33.61\right)$, BP $\left(P<0.001 ; \mathrm{F}_{2,27}=\right.$ 46.91), and $\log \mathrm{Koa}\left(P<0.001 ; \mathrm{F}_{2,27}=30.63\right)$, and a tendency for $\mathrm{HC}\left(P<0.0054 ; \mathrm{F}_{2,27}=6.48\right)$ and VP $\left(P<0.0094 ; \mathrm{F}_{2,27}=5.65\right.$; Fig. 3). Fungicides were characterized by higher values of MW, PS, BP, and log Koa compared with insecticidal and attractive-toinsects compounds, but no significant differences were observed between insecticidal and attractive compounds (significant differences between pairs determined by Tukey's HSD tests). Concerning HC and VP, attractive compounds exhibited higher values than fungicides and insecticides.

Fungitoxic compounds tested in this study are characterized by significantly higher log Kow than compounds with no fungitoxic activities (mean fungitoxic: 2.41 ; mean not fungitoxic: $0.82 ; P=$ $0.0049, \mathrm{~F}_{1,49}=8.69$ ). Hence, fungitoxic compounds exhibit higher bioaccumulation potential and are more lipophilic than compounds with no fungitoxic activities.

\section{Physicochemical properties of antifungal GLS derivatives versus other natural and synthetic fungicides}

The PCA revealed differences in physicochemical characteristics between synthetic and natural fungicides from plant, algal, bacterial, fungal, and poriferan origins (Fig. 4). Allyl isothiocyanate GLSD was removed from the analyses because it was detected as an outlier from the outlier analyses $\left(\mathrm{T}^{2}>120\right.$; overall 
median $\mathrm{T}^{2}=7.36$ ). It is indeed a very small and highly volatile molecule compared with other fungitoxic compounds. The two first principal components explained $72.1 \%$ of the variance (PC1: 46.5\%; PC2: 25.6\%; Fig. 4). Quadrant I represents large hydrophilic molecules with high bioaccumulation potential (mainly from bacteria and porifera), quadrant II large hydrophobic molecules with the capacity to go through membranes and high bioaccumulation potential (from synthetic, poriferan, and fungi origins), quadrant III small hydrophobic compounds with the capacity to go through membranes (mainly synthetic compounds and from plant origin [GLSD and non-GLSD]), and quadrant IV small more volatile and hydrophilic ones (from synthetic, plant [GLSD and non-GLSD], and fungi origins). Hence, GLSD (except allyl isothiocyanate) cluster with non-GLSD fungicides from plant origins as well as synthetic compounds and few compounds from fungal origins in quadrants III and IV.

After Bonferroni correction (significance level: 0.00625), ANOVAs revealed overall significant differences between compounds from different origins (GLSD, plant and algae, fungi, porifera, bacteria, and synthetic $)$ for $\mathrm{MW}\left(P<0.001 ; \mathrm{F}_{5,132}=\right.$ 26.2), BP $\left(P<0.001 ; \mathrm{F}_{5,132}=34.1\right)$, PS $\left(P<0.001 ; \mathrm{F}_{5,132}=37.3\right)$, VP $\left(P<0.0021 ; \mathrm{F}_{5,132}=4.0\right), \mathrm{HC}\left(P<0.004 ; \mathrm{F}_{5,132}=3.7\right), \log$ Koa $\left(P<0.001 ; \mathrm{F}_{5,132}=26.4\right), \log$ Kow $\left(P<0.001 ; \mathrm{F}_{5,132}=\right.$ 19.1), and $\log \mathrm{S}\left(P<0.001 ; \mathrm{F}_{5,132}=11.1\right)$ (Fig. 5). No significant differences were observed between GLSD and non-GLSD compounds from plants for all these variables, except for VP and HC with non-GLSD compounds (VP: 0.011; HC: 0.00166) being more volatile than GLSD (VP: 0.00031; HC: 0.00002; significant differences between pairs determined by Tukey's HSD tests; Fig. 5). Similarly, no differences were observed between synthetic fungicides and GLSD, except for log $\mathrm{S}$ (mean synthetic: -4.33 , range: -7.6 to -1.2 ; mean GLSD: -2.46 , range: -3.3 to -1.0 ).

Significant differences in log Kow are the result of differences between fungicides from bacterial (genus Streptomyces; primarily described and used as antibiotic but also showing fungitoxic activities) and other origins (log Kow bacteria mean: -4.92, log Kow other origins mean: 2.78).

\section{DISCUSSION}

Following up on our recent study highlighting that a breakdown product of 7-methylsulfonylheptyl glucosinolate (compound 1), which overaccumulated on the cotyledon of Arabidopsis thaliana PUFA mutants, has antifungal properties (Dubey et al. 2020), here we confirm that 13 out of 31 tested natural and semisynthetic methylsulfonyl and methylsulfinyl isothiocyanates are efficient fungicides alone (seven) and/or when used in combination against widespread genetically distant species of plant pathogens. Overall, 17 combinations of these 13 compounds showed strong synergistic fungitoxic effects (all $\mathrm{CI}<0.3$; Chou 2008) on the three tested fungal pathogens, the strongest observed synergy being when compounds 12 and 13 are combined (A. radicina; EC50 compound $12=723 \mu \mathrm{M}$; EC50 compound $13>850 \mu \mathrm{M}$; EC50 combined $=156 \mu \mathrm{M}$; CI = 0.029). Importantly, only the combination of compounds 1 and 9 was fungicidal against all three species, whereas others were only for one or two of the tested species, with $F$. graminearum being much more susceptible to GLSD than $A$. radicina and $P$. cucumerina. These results highlight that methylsulfonyl and methylsulfinyl isothiocyanate GLSD are more efficient when combined and that their mode of action might differ depending on the structure of the compounds. It might in turn explain why some combinations are species specific.

Interestingly, physicochemical characteristics of fungitoxic GLSD differ from those showing no activities (this study) or from those that are known for their insecticidal or insect-attractive properties (Lambrix et al. 2001; Wittstock et al. 2003) (see Fig. 2 and discriminant analysis). Indeed, fungitoxic compounds exhibit significantly higher bioaccumulation potential and are more lipophilic (log Kow: 2.41) than compounds with no fungitoxic activities (log Kow: 0.82).

Overall, GLSD with fungitoxic properties are characterized by higher capacity to permeate cells (PS) and a higher bioaccumulation potential (log Koa) than GLSD with insecticidal and attractive properties (Fig. 3). In addition, fungitoxic GLSD are less volatile than insecticidal and attractive GLSD. It is

\section{TABLE 1}

Best combinations of molecules against Alternaria radicina, Fusarium graminearum, and Plectosphaerella cucumerina. Molecules for which $>850$ is mentioned for EC50 are those with no fungitoxic activity in the tested range. Combination index (CI) shows presence of synergism when CI $<1$, with values between 0.1 and 0.3 considered as strong synergism and values $<0.1$ as very strong synergism.

\begin{tabular}{|c|c|c|c|c|c|}
\hline Species & Combination & EC50 molecule $1(\mu \mathrm{M})$ & EC50 molecule $2(\mu \mathrm{M})$ & EC50 combination $(\mu \mathrm{M})$ & $\mathrm{CI}$ \\
\hline A. radicina & 8ASOH/8ASOOH & 723 & $>850$ & 156 & 0.02872 \\
\hline A. radicina & $8 \mathrm{CSOH} / 8 \mathrm{ESOH}$ & 704 & 472 & 390 & 0.08735 \\
\hline A. radicina & $8 \mathrm{CSOH} / 8 \mathrm{ESOOH}$ & 472 & $>850$ & 474 & 0.115 \\
\hline A. radicina & $8 \mathrm{CSOH} / 3 \mathrm{MSOOH}$ & 704 & 469 & 479 & 0.02991 \\
\hline A. radicina & $8 \mathrm{CSOH} / 7 \mathrm{MSOH}$ & 704 & 537 & 534 & 0.08249 \\
\hline A. radicina & $8 \mathrm{CSOH} / 3 \mathrm{MSOH}$ & 704 & 786 & 626 & 0.25268 \\
\hline F. graminearum & $8 \mathrm{MSOH} / 3 \mathrm{MSOOH}$ & 317 & 387 & 252 & 0.24857 \\
\hline F. graminearum & $8 \mathrm{MSOH} / 7 \mathrm{MSOH}$ & 317 & 586 & 258 & 0.16426 \\
\hline F. graminearum & 8MSOH/9MSOH & 317 & 553 & 263 & 0.15388 \\
\hline F. graminearum & $8 \mathrm{MSOH} / 6 \mathrm{MSOH}$ & 317 & $>850$ & 266 & 0.25732 \\
\hline F. graminearum & 8CSOH/8MSOH & $>850$ & 317 & 275 & 0.13763 \\
\hline F. graminearum & 8CSOH/3MSOOH & $>850$ & 387 & 282 & 0.10929 \\
\hline$F$. graminearum & 8MSOH/9MSOOH & 317 & $>850$ & 284 & 0.13736 \\
\hline F. graminearum & $8 \mathrm{CSOH} / 3 \mathrm{MSOH}$ & $>850$ & 525 & 384 & 0.04368 \\
\hline F. graminearum & $8 \mathrm{CSOH} / 7 \mathrm{MSOH}$ & $>850$ & 586 & 389 & 0.03555 \\
\hline F. graminearum & $8 \mathrm{ESOH} / 7 \mathrm{MSOH}$ & 533 & 586 & 414 & 0.12419 \\
\hline F. graminearum & $8 \mathrm{ESOH} / 3 \mathrm{MSOH}$ & 533 & 525 & 455 & 0.15703 \\
\hline F. graminearum & 8MSOOH/7MSOH & $>850$ & 586 & 541 & 0.06049 \\
\hline P. cucumerina & $8 \mathrm{CSOH} / 7 \mathrm{MSOH}$ & 273 & 337 & 146 & 0.18832 \\
\hline P. cucumerina & 8MSOOH/3MSOH & $>850$ & 627 & 483 & 0.09631 \\
\hline P. cucumerina & $8 \mathrm{MSOOH} / 3 \mathrm{MSOOH}$ & $>850$ & 536 & 521 & 0.03061 \\
\hline
\end{tabular}



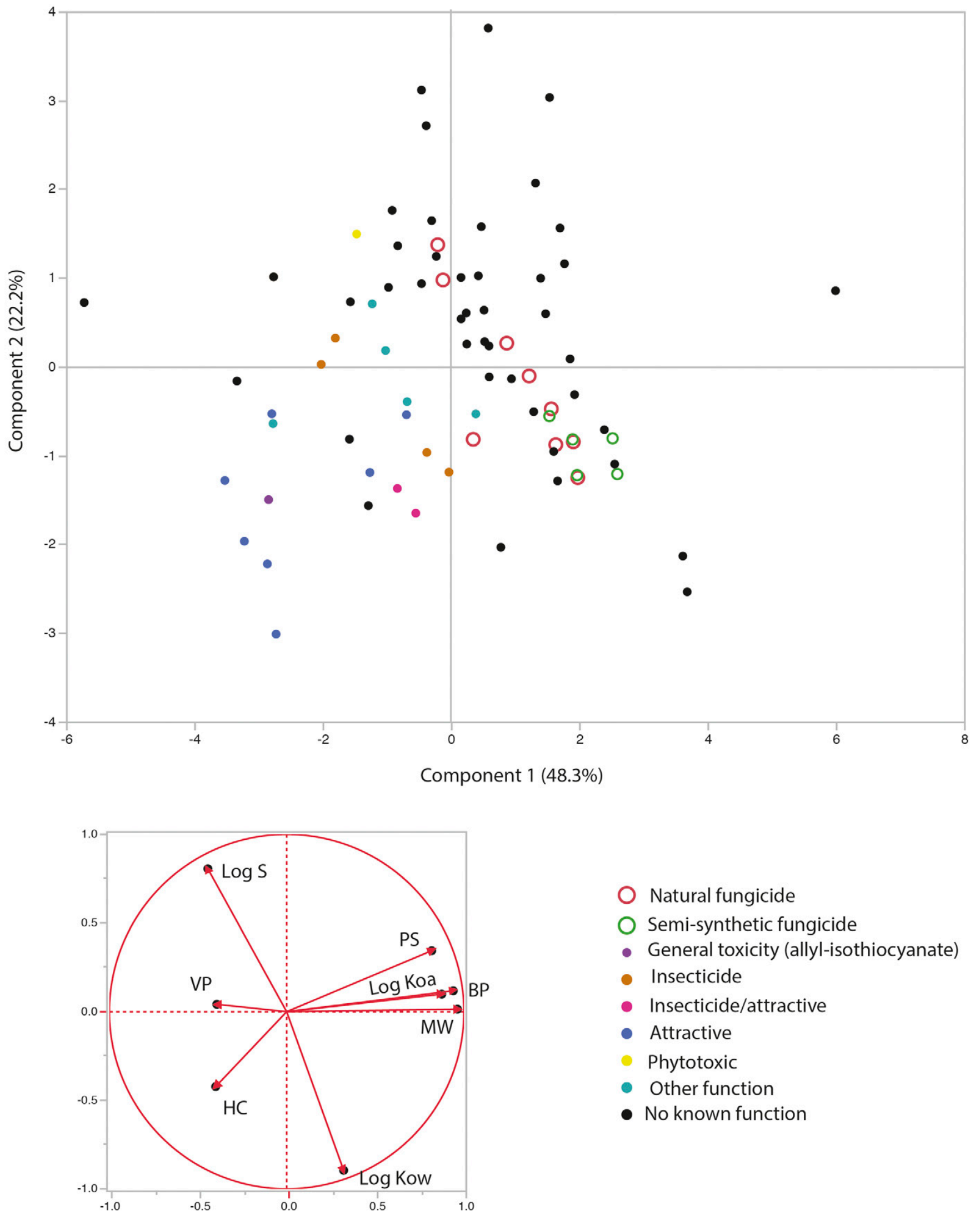

Natural fungicide

Semi-synthetic fungicide

- General toxicity (allyl-isothiocyanate)

- Insecticide

- Insecticide/attractive

- Attractive

- Phytotoxic

- Other function

- No known function

FIGURE 2

Principal component analysis on eight physicochemical variables in glucosinolate derivatives (GLSD). Their contributions to the first two axes displayed are indicated by vectors as follows: log of intrinsic solubility in mol/liter (log S), log octanol/water partition coefficient (log Kow), log octanol/air partition coefficient (log Koa), boiling point (BP), polar surface area (PS), molecular weight (MW), vapor pressure (VP), and Henry constant (HC). The known function of GLSD is indicated by colors as follows: red open circle = natural fungicide; green open circle = semisynthetic fungicide; violet = general toxicity; brown = insecticide; pink = insecticide and attractive; blue = attractive; yellow $=$ phytotoxic; turquoise $=$ other functions; and black = no known function. 
therefore possible to anticipate the potential function of GLSD based on their physicochemical properties and the differences in the way compounds will interact with the surrounding environment and hence organisms. Observed fungitoxic synergistic effects of GLSD (Table 1) suggest that the mode of action between these compounds might to some extent differ between themselves. This hypothesis is also in line with the fact that differences in the efficacy of combinations are observed on the tested fungal pathogens, some being efficient only on one pathogen.
Previous studies revealed that isothiocyanates are toxic not only to fungi but also to insects, bacteria, and humans, likely due to their mode of action (react with amino groups of proteins and cleave disulfide bonds in vitro (Kawakishi and Kaneko 1985, 1987; Wittstock et al. 2003). They also revealed that these compounds are highly mutagenic (as revealed by positive Ames tests-reverse mutation assays; e.g., National Institute of Environmental Health Sciences 1981a, 1981b; Neudecker and Henschler 1985), making them unsuitable for use as pesticides. However, those studies focused on allyl isothiocyanate, a highly

\section{FIGURE 3}

Distribution (median with standard error and $95 \%$ standard deviation) of molecular weight (MW), polar surface area (PS), boiling point $(\mathrm{BP})$, and log Koa in (natural and semisynthetic) glucosinolate derivatives from this study and previously published data. Letters indicate statistically significant differences between pairs as determined by Tukey's HSD test.

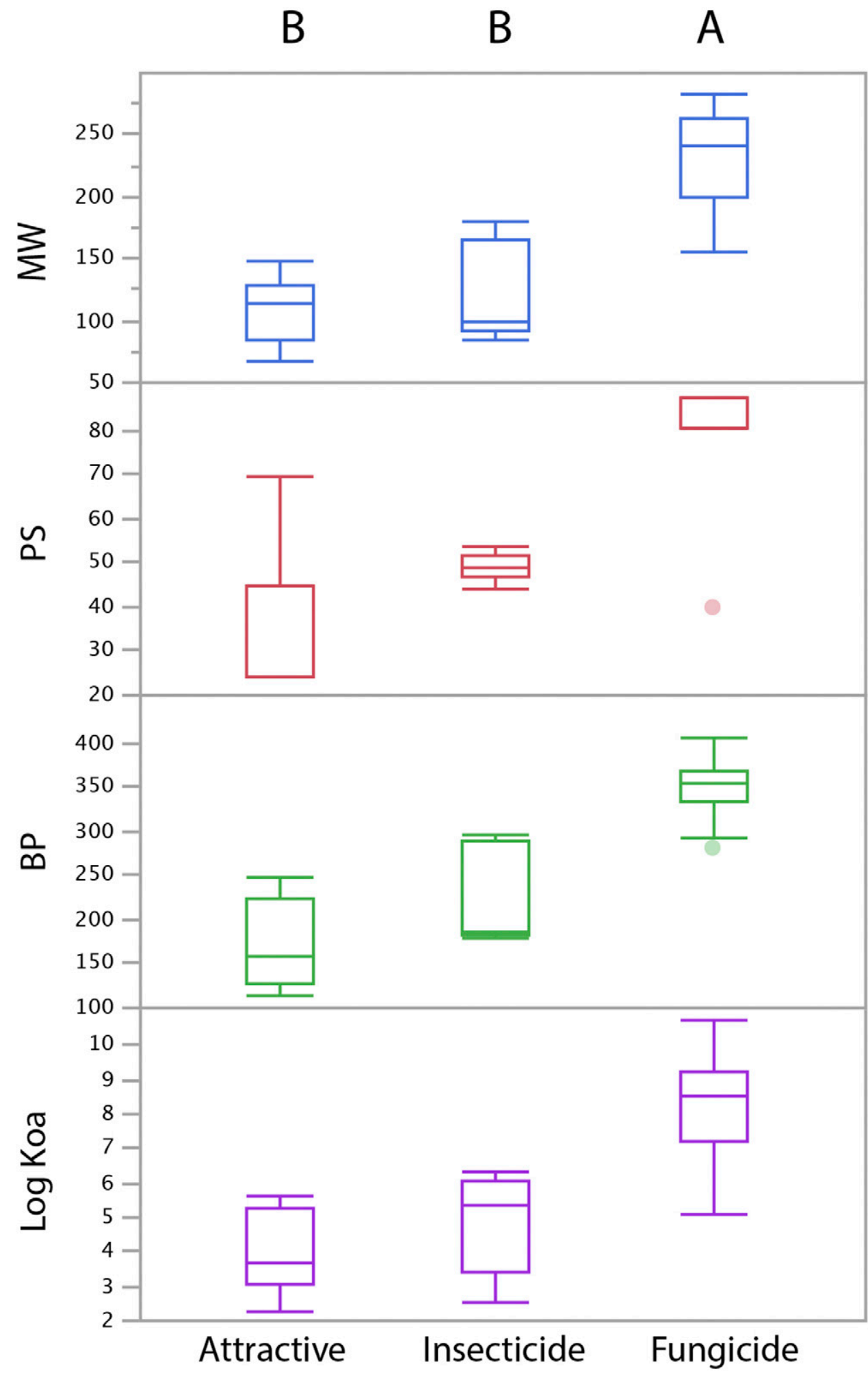


volatile compound, and not on methylsulfonyl and methylsulfinyl isothiocyanates (this study), which exhibit very different physicochemical properties, and hence allyl isothiocyanate cannot be compared with methylsulfinyl isothiocyanates. These assumptions are fitting with Ames tests performed on two compounds from the present study (compounds 5 and 6; Ames test study numbers performed by Envigo CRS GmbH: 1936801 and 1936802), which revealed no mutagenicity effects on Salmonella typhimurium and
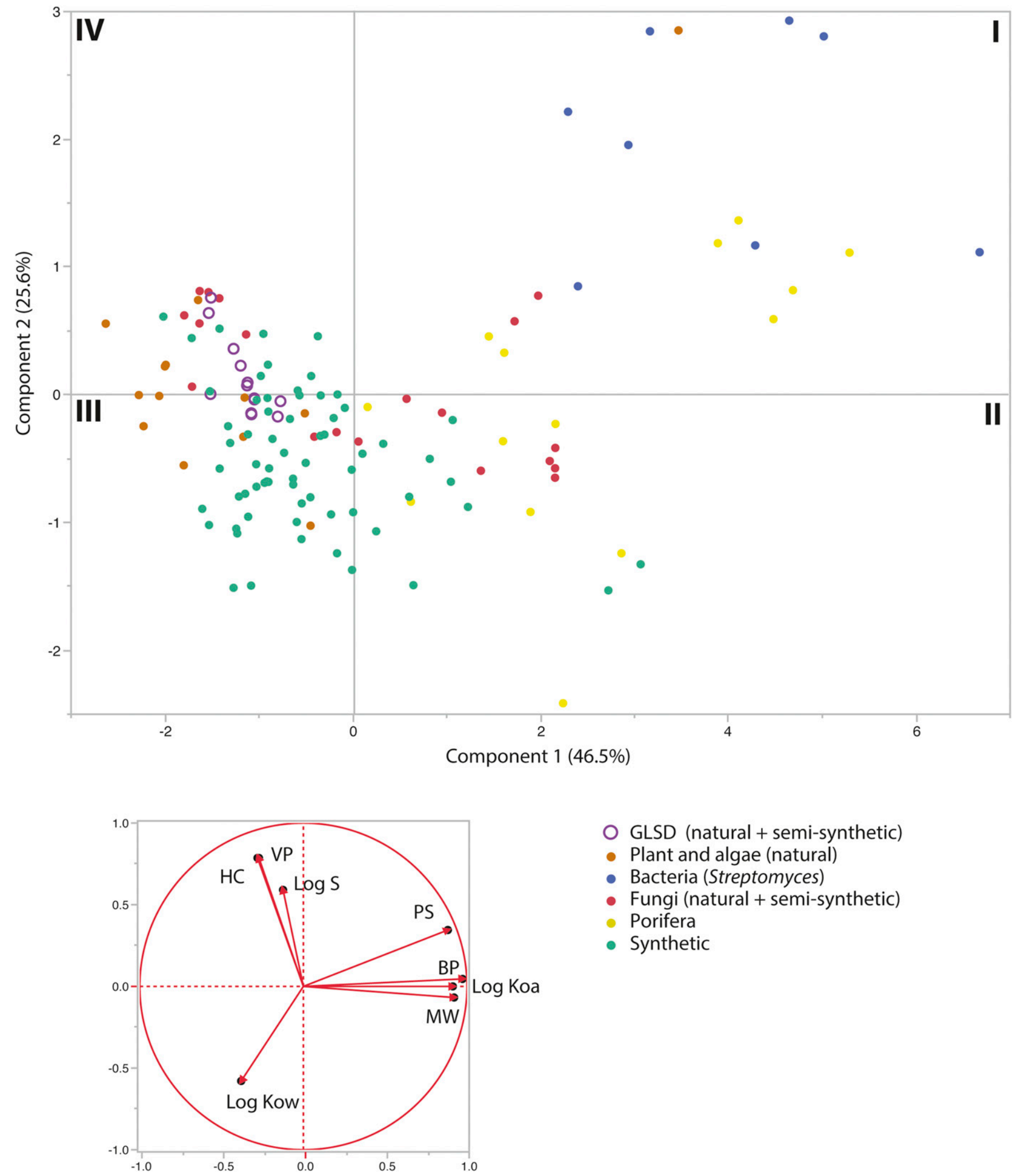

O GLSD (natural + semi-synthetic)

- Plant and algae (natural)

- Bacteria (Streptomyces)

- Fungi (natural + semi-synthetic)

- Porifera

- Synthetic

FIGURE 4

Principal component analysis on eight physicochemical variables in fungicides. Their contributions to the first two axes displayed are indicated by vectors as follows: log of intrinsic solubility in mol/liter (log S), log octanol/water partition coefficient (log Kow), log octanol/air partition coefficient (log Koa), boiling point (BP), polar surface area (PS), molecular weight (MW), vapor pressure (VP), and Henry constant $(\mathrm{HC})$. The quadrants are numbered clockwise starting upper right, and the origin of fungicide is indicated by colors as follows: open violet circle = glucosinolate derivatives (GLSD); brown = plant and algae (non-GLSD); blue = bacteria; red = fungi; yellow = porifera; and green $=$ synthetic. 
Escherichia coli even at high concentrations (both with and without liver microsomal activation).

The comparison of physicochemical characteristics of natural and semisynthetic fungitoxic GLSD with other fungicides (natural, semisynthetic, and synthetic) revealed that isothiocyanate GLSD (except allyl isothiocyanate) clustered with natural fungicides from plants (non-GLSD: i.e., resveratrol, dehydro- $\alpha$-lapachone, and 4acetyltropolone) and fungi (sphaeropsidone, afritoxinone A, oxysporone, and $(3 R, 4 R)$-4-hydroxymellein), as well as with some synthetic ones (fluoroimide, dichloran, fentin acetate, prothiocarb, and flucytosine) in the PCA (quadrants III and IV; Fig. 4). Within fungicides, these compounds are characterized by small MW, low $\mathrm{BP}$, and small accumulation potential compared with fungicides from the other quadrants, particularly quadrant I, which includes mainly compounds from fungi and porifera characterized by large MW and BP as well as very high log Koa. However, overall and independently of their origin, fungicides show high bioaccumulation potential (i.e., mean $\log$ Koa $>8$; see Swanson and Vighi 1998) and are lipophilic; hence, they have the capacity to go through membranes (mean log Kow: 2.3), which seems to be a

\section{FIGURE 5}

Distribution (median with standard error and 95\% standard deviation) of molecular weight (MW), log of intrinsic solubility in mol/liter (log S), log octanol/water partition coefficient (log Kow), log octanol/air partition coefficient (log Koa), polar surface area (PS), boiling point (BP), vapor pressure (VP), and Henry constant (HC) in fungicides from bacteria, fungi, porifera, plant glucosinolate derivatives (plant GLSD), plant and algae (non-GLSD), and synthetic compounds from this study and previously published data. Letters indicate statistically significant differences between pairs as determined by Tukey's HSD test.

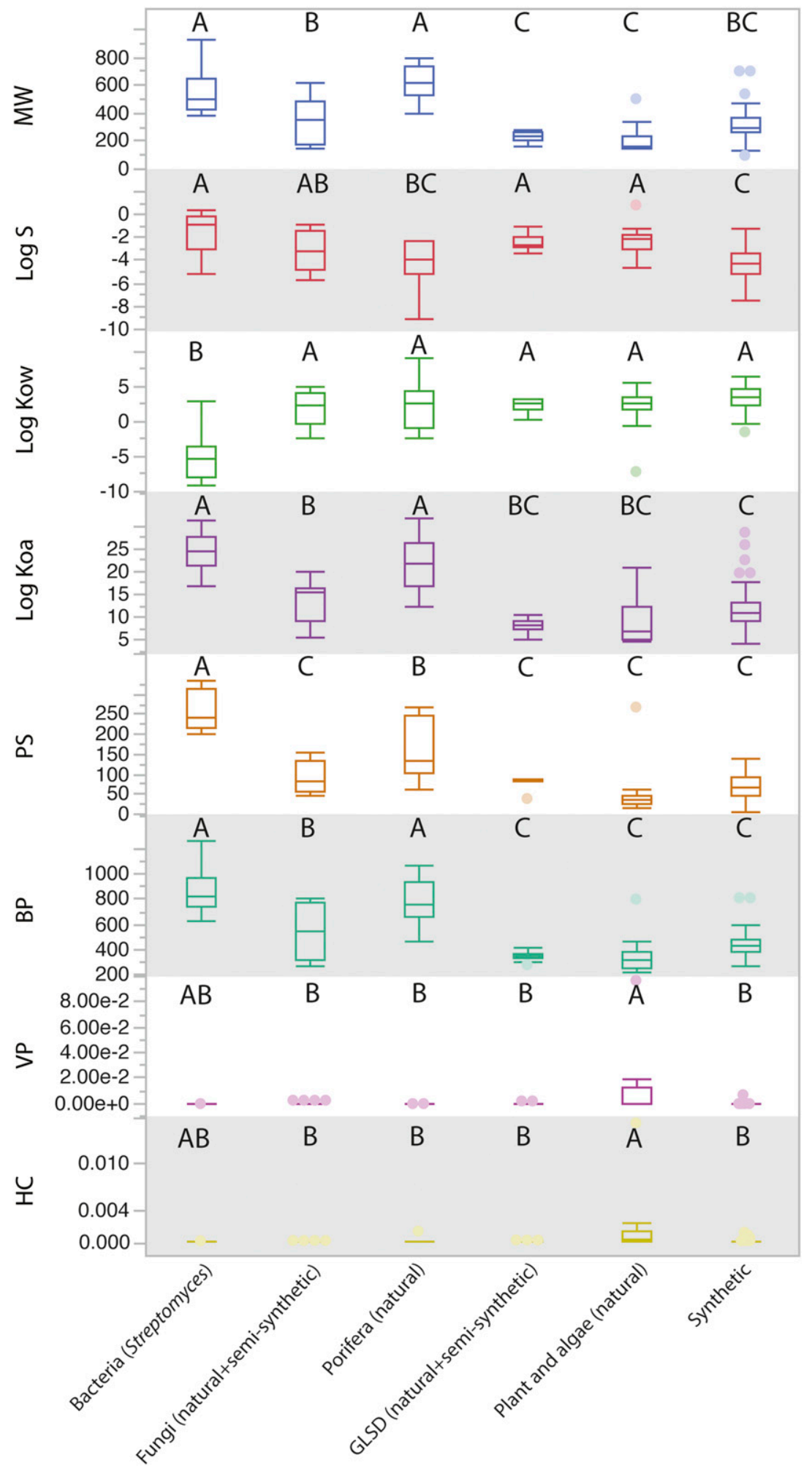


prerequisite for fungitoxic activities. According to Schröder and Collins (2010), fungicides are considered systemic when applied on plants if $\log$ Kow < 4; more specifically, it is xylem mobile only when $\log$ Kow is situated between 0 and 4 and phloem and xylem mobile when $\log$ Kow is situated between 0 and -3 . Hence, fungitoxic GLSD described in the present study can be considered as systemic and xylem mobile (mean log Kow: 2.41). Systemic fungicides were first described by Von Schmeling and Kulka (1966) and since then have offered new opportunities in disease control compared with contact ones, which remain on the surface of the plants and hence have limited modes of action (Russell 2005). Indeed, such fungicides are able to stop pathogens from developing after the infection process has already started, by fighting the pathogen directly from the inside of plants (Russell 2005).

On the other hand, compounds from bacterial origins (widespread genus Streptomyces), primarily described and used as antibiotics but also showing fungitoxic activities (mode of action: amino acids and protein synthesis), are hydrophilic with a low capacity to go through membranes (mean log Kow: -4.92), characteristics that strongly differ with compounds from other origins. This property is shared by antibiotics with human and veterinary usage such as penicillin $\mathrm{G}$ and tetracycline, for example (Gin and $\mathrm{Ng} 2019$ ). In order to target intracellular processes, antibiotics need to penetrate the envelope of cells. It can be achieved through a lipid-mediated pathway for hydrophobic antibiotics (penetration of the outer membrane) or by general diffusion porins for hydrophilic ones (Delcour 2009; Nikaido 2003). Interestingly, allyl isothiocyanate, the fungitoxic GLSD presenting a general toxicity to living organisms, is also presenting unique atypical properties (high volatility, lowest MW of analyzed compounds, and low bioaccumulation [log Koa]).

Resveratrol, a well-described fungitoxic phytoalexin synthetized by plants in response to external stresses, exhibits physicochemical characteristics close to GLSD (Fig. 4; Caruso et al. 2011). Interestingly, it shares additional properties with GLSD, such as antioxidant and antitumor activities, and both are present in a large number of plant species (Chang et al. 2011; De Figueiredo et al. 2013; Salehi et al. 2018). The fungitoxic mode of action of resveratrol is not well understood, and it is classified as a host plant defense induction fungicide (class P5; FRAC [2019] database), but transmission electron microscopy analyses showed that it is damaging the conidia of Botrytis cinerea (gray mold; Adrian and Jeandet 2012). Fungitoxic activities are not only known against this species but also for a wide range of fungi and yeast: Trichophyton spp., Epidermophyton floccosum, Microsporum gypseum, Saccharomyces cerevisiae, and Trichosporon beigelii (Vestergaard and Ingmer 2019). Hence, it exhibits a broad spectrum of activities as isothiocyanate GLSD (this study; Dubey et al. 2020).

Oxidation products of resveratrol (viniferins), probably induced by peroxidases in plants, are even more toxic (Pezet et al. 2004). Because fungi such as $B$. cinerea can also transform resveratrol in viniferin with a stilbene oxidase, in vitro fungitoxic activities of resveratrol described above could be explained by the production of viniferin (Pezet et al. 2004; Schouten et al. 2002). Concerning isothiocyanate GLSD, Dubey et al. (2020) showed that compound 1 is impacting the vacuole of $B$. cinerea, but further analyses are required to understand the mode of action. We cannot exclude that fungal pathogens are transforming GLSD through similar enzymatic reactions into more fungitoxic compounds. However, such information is not yet available. In synthetic fungicides, modes of action are better documented, and synthetic ones clustering with GLSD are impacting, for example, the lipid synthesis or transport and membrane integrity or function (dichloran and prothiocarb), the synthesis of both DNA and RNA (flucytosine), or the respiration (fentin acetate), or they are exhibiting multiple-site activities (fluoroimide; FRAC 2019; MacManus 2015).

In conclusion, this study revealed that methylsulfonyl and methylsulfinyl isothiocyanate GLSD are a source of fungitoxic compounds that exhibit a broad spectrum of activities with physicochemical properties similar to natural and synthetic fungicides and that, apart from allyl isothiocyanate, seem to show no mutagenic properties preventing their use based on the Ames tests performed on compounds 5 and 6 .

\section{ACKNOWLEDGMENTS}

We thank J. Schuerch and R. Dubey for helpful comments and interesting discussions.

\section{LITERATURE CITED}

Adrian, M., and Jeandet, P. 2012. Effects of resveratrol on the ultrastructure of Botrytis cinerea conidia and biological significance in plant/pathogen interactions. Fitoterapia 83:1345-1350.

Agerbirk, N., and Olsen, C. E. 2012. Glucosinolate structures in evolution. Phytochemistry 77:16-45.

Ahmad, A., Khan, A., Khan, L. A., and Manzoor, N. 2010. In vitro synergy of eugenol and methyleugenol with fluconazole against clinical Candida isolates. J. Med. Microbiol. 59:1178-1184.

Blažević, I., Montaut, S., Burčul, F., Olsen, C. E., Burow, M., Rollin, P., and Agerbirk, N. 2020. Glucosinolate structural diversity, identification, chemical synthesis and metabolism in plants. Phytochemistry 169:112100.

Cartea, M. E., and Velasco, P. 2008. Glucosinolates in Brassica foods: Bioavailability in food and significance for human health. Phytochem. Rev. 7:213-229.

Caruso, F., Mendoza, L., Castro, P., Cotoras, M., Aguirre, M., Matsuhiro, B., Isaacs, M., Rossi, M., Viglianti, A., and Antonioletti, R. 2011. Antifungal activity of resveratrol against Botrytis cinerea is improved using 2-furyl derivatives. PLoS One 6:e25421.

Chang, X., Heene, E., Qiao, F., and Nick, P. 2011. The phytoalexin resveratrol regulates the initiation of hypersensitive cell death in Vitis cell. PLoS One 6:e26405.

Chhajed, S., Misra, B. B., Tello, N., and Chen, S. 2019. Chemodiversity of the glucosinolate-myrosinase system at the single cell type resolution. Front. Plant Sci. 10:618.

Chou, T. C. 2006. Theoretical basis, experimental design, and computerized simulation of synergism and antagonism in drug combination studies. Pharmacol. Rev. 58:621-681.

Chou, T. C. 2008. Preclinical versus clinical drug combination studies. Leuk. Lymphoma 49:2059-2080.

Chou, T. C., and Martin, N. 2005. CompuSyn for drug combinations: PC software and user's guide: A Computer Program for quantitation of synergism and antagonism in drug combinations, and the determination of IC50 and ED50 and LD50 values. ComboSyn, Paramus, NJ.

Cortés-Rojas, D. F., Fernandes de Souza, C. R., and Oliveira, W. P. 2014. Clove (Syzygium aromaticum): A precious spice. Asian Pac. J. Trop. Biomed. 4:90-96.

De Figueiredo, S. M., Filho, S. A. V., Nogueira-Machado, J. A., and Caligiorne, R. B. 2013. The anti-oxidant properties of isothiocyanates: A review. Recent Pat. Endocr. Metab. Immune Drug Discov. 7:213-225.

Delcour, A. H. 2009. Outer membrane permeability and antibiotic resistance. Biochim. Biophys. Acta 1794:808-816.

Dubey, O., Dubey, S., Schnee, S., Glauser, G., Nawrath, C., Gindro, K., and Farmer, E. E. 2020. Plant surface metabolites as potent antifungal agents. Plant Physiol. Biochem. 150:39-48.

Dufour, V., Stahl, M., and Baysse, C. 2015. The antibacterial properties of isothiocyanates. Microbiology-Sgm. 161:229-243.

El Amraoui, B., El Wahidi, M., and Fassouane, A. 2014. In vitro screening of antifungal activity of marine sponge extracts against five phytopathogenic fungi. SpringerPlus 3:629.

Fahey, J. W., Zalcmann, A. T., and Talalay, P. 2001. The chemical diversity and distribution of glucosinolates and isothiocyanates among plants. Phytochemistry 56:5-51.

FAO. 2011. Global Food Losses and Food Waste: Extent, Causes and Prevention. FAO, Rome, Italy.

FRAC. 2019. https://www.frac.info/. 
Gin, K. Y. H., and Ng, C. 2019. Antimicrobial Resistance in Environmental Waters. MDPI, Basel, Switzerland.

Gonzalez-Fernandez, R., Prats, E., and Jorrin-Novo, J. V. 2010. Proteomics of plant pathogenic fungi. J. Biomed. Biotechnol. 1:36.

Hadizadeh, S., Forootanfar, H., Bonjar, G. H. S., Nejad, M. F., Robati, A. K., Mousavi, S. A. A., and Amirporrostami, S. 2015. Antifungal activity of terrestrial Streptomyces rochei strain HF391 against clinical azole -resistant Aspergillus fumigatus. Curr. Med. Mycol. 1:19-24.

Hanschen, F. S., Klopsch, R., Oliviero, T., Schreiner, M., Verkerk, R., and Dekker, M. 2017. Optimizing isothiocyanate formation during enzymatic glucosinolate breakdown by adjusting $\mathrm{pH}$ value, temperature and dilution in Brassica vegetables and Arabidopsis thaliana. Sci. Rep. 7:40807.

Ishida, M., Hara, M., Fukino, N., Kakizaki, T., and Morimitsu, Y. 2014. Glucosinolate metabolism, functionality and breeding for the improvement of Brassicaceae vegetables. Breed. Sci. 64:48-59.

Kawakishi, S., and Kaneko, T. 1985. Interaction of oxidized glutathione with allyl isothiocyanate. Phytochemistry 24:715-718.

Kawakishi, S., and Kaneko, T. 1987. Interaction of proteins with allyl isothiocyanate. J. Agric. Food Chem. 35:85-88.

Kos, M., Houshyani, B., Achhami, B. B., Wietsma, R., Gols, R., Weldegergis, B. T., Kabouw, P., Bouwmeester, H. J., Vet, L. E. M., Dicke, M., and van Loon, J. J. A. 2012. Herbivore-mediated effects of glucosinolates on different natural enemies of a specialist aphid. J. Chem. Ecol. 38:100-115.

Lambrix, V., Reichelt, M., Mitchell-Olds, T., Kliebenstein, D. J., and Gershenzon, J. 2001. The Arabidopsis epithiospecifier protein promotes the hydrolysis of glucosinolates to nitriles and influences Trichoplusia ni herbivory. Plant Cell 13:2793-2807.

Lucas, J. A., Hawkins, N. J., and Fraaij, B. A. 2015. The evolution of fungicide resistance. Adv. Appl. Microbiol. 90:29-92.

MacManus, D. S. 2015. Antifungal drugs. Side Eff. Drugs Annu. 37:307-319.

National Institute of Environmental Health Sciences. 1981a. NTP study results. Genetic toxicity evaluation of allyl isothiocyanate in Salmonellal E.coli mutagenicity test or Ames test. Study 521783.

National Institute of Environmental Health Sciences. 1981b. NTP study results. Genetic toxicity evaluation of allyl isothiocyanate in Salmonellal E.coli mutagenicity test or Ames test. Study 649249.

Neudecker, and T., Henschler, D. 1985. Allyl isothiocyanate is mutagenic in Salmonella typhimurium. Mutat. Res., Genet. Toxicol. 156:33-37.

Nikaido, H. 2003. Molecular basis of bacterial outer membrane permeability revisited. Microbiol. Mol. Biol. Rev. 67:593-656.

Pezet, R., Viret, O., and Gindro, K. 2004. Plant-microbe interaction: The Botrytis Grey mould of grapes-biology, biochemistry, epidemiology and control management. Adv. Plant Physiol. 7:71-116.

Rice, W. 1989. Analyzing tables of statistical tests. Evolution 43:223-225. Russell, P. E. 2005. A century of fungicide evolution. J. Agric. Sci. 143:11-25.
Salehi, B., Mishra, A. P., Nigam, M., Sener, B., Kilic, M., Sharifi-Rad, M. Fokou, P. V. T., Martins, N., and Sharifi-Rad, J. 2018. Resveratrol: A double-edged sword in health benefits. Biomedicines 6:91.

Schouten, A., Wagemakers, L., Stefanato, F. L., van der Kaaij, R. M., and van Kan, J. A. L. 2002. Resveratrol acts as a natural profungicide and induces self-intoxication by a specific laccase. Mol. Microbiol. 43: 883-894.

Schröder, P., and Collins, C. D. 2010. Organic Xenobiotics and Plants. Springer Science \& Business Media, Berlin, Germany.

Swanson, T. M., and Vighi, M., eds. 1998. Regulating Chemical Accumulation in the Environment: The Integration of Toxicology and Economics in Environmental Policy-Making. Cambridge University Press, Cambridge, U.K

Tang, X., Shao, Y. L., Tang, Y. J., and Zhou, W. W. 2018. Antifungal activity of essential oil compounds (geraniol and citral) and inhibitory mechanisms on grain pathogens (Aspergillus flavus and Aspergillus ochraceus). Molecules 23:2108.

U.S. EPA. 2020. Estimation Programs Interface Suite ${ }^{\mathrm{TM}}$ for Microsoft ${ }^{\circledR}$ Windows, version 4.11. United States Environmental Protection Agency, Washington, DC.

van Poecke, R. M. P., Posthunus, A., and Dicke, M. 2001. Herbivore-induced volatile production by Arabidopsis thaliana leads to attraction of the parasitoid Cotesia rubecula: Chemical, behavioral, and gene-expression analysis. J. Chem. Ecol. 27:1911-1928.

Vanhaelen, N., Gaspar, C., and Francis, F. 2002. Influence of prey host plant on a generalist aphidophagous predator: Episyrphus balteatus (Diptera: Syrphidae). Eur. J. Entomol. 99:561-564.

Vestergaard, M., and Ingmer, H. 2019. Antibacterial and antifungal properties of resveratrol. Int. J. Antimicrob. Agents 53:716-723.

Von Schmeling, B., and Kulka, M. 1966. Systemic fungicidal activity of 1,4 oxathiin derivatives. Science 152:659-660.

Wittstock, U., Kliebenstein, D. J., Lambrix, V., Reichelt, M., and Gershenzon, J. 2003. Glucosinolate hydrolysis and its impact on generalist and specialist insect herbivores. Recent Adv. Phytochem. 37: 101-125.

Yamane, H., Konno, K., Sabelis, M., Takabayashi, J., Sassa, T., and Oikawa, H. 2010. Pages 339-385 in: Comprehensive Natural Products II. Chemistry and Biology. Vol. 4. L. Mander and H. W. Lui, eds. Elsevier, Oxford, U.K.

Yoon, M. Y., Cha, B., and Kim, J. C. 2013. Recent trends in studies on botanical fungicides in agriculture. Plant Pathol. J. 29:1-9.

Yoon, M. Y., Choi, N. H., Min, B. S., Choi, G. J., Choi, Y. H., Jang, K. S., Han, S. S., Cha, B., and Kim, J. C. 2011. Potent in vivo antifungal activity against powdery mildews of pregnane glycosides from the roots of Cynanchum wilfordii. J. Agric. Food Chem. 59:12210-12216. 